\title{
Application of velocity-based gain-scheduling to lateral auto-pilot design for an agile missile
}

\author{
D.J. Leith ${ }^{\mathrm{a}, *}$, A. Tsourdos ${ }^{\mathrm{b}}$, B.A. White ${ }^{\mathrm{b}}$, W.E. Leithead ${ }^{\mathrm{a}}$ \\ ${ }^{a}$ Department of Electronic \& Electrical Engineering, University of Strathclyde, 50 George Street, Glasgow G1 1QE, UK \\ ${ }^{\mathrm{b}}$ Department of Aerospace, Power \& Sensors, Cranfield University-RMCS, Swindon SN6 8LA, UK
}

Received 9 April 2001; accepted 9 April 2001

\begin{abstract}
In this paper a modern gain-scheduling methodology is proposed which exploits recently developed velocity-based techniques to resolve many of the deficiencies of classical gain-scheduling approaches (restriction to near equilibrium operation, to slow rate of variation). This is achieved while maintaining continuity with linear methods and providing an open design framework (any linear synthesis approach may be used) which supports divide and conquer design strategies. The application of velocity-based gainscheduling techniques is demonstrated in application to a demanding, highly nonlinear, missile control design task. Scheduling on instantaneous incidence (a rapidly varying quantity) is well-known to lead to considerable difficulties with classical gain-scheduling methods. It is shown that the methods proposed here can, however, be used to successfully design an effective and robust gainscheduled controller. (C) 2001 Elsevier Science Ltd. All rights reserved.
\end{abstract}

\section{Introduction}

Gain-scheduling control is widely employed in flight control applications, where high performance has to be achieved over a broad operating envelope. In the classical gain-scheduling design approach, a nonlinear controller is constructed by continuously interpolating, in some manner, between the members of a family of linear controllers (see, for example, the recent survey of Leith \& Leithead, 2000a). Each linear controller is, typically, associated with a specific equilibrium operating point of the plant and is designed to ensure that, locally to the equilibrium operating point, the performance requirements are met. By employing a series expansion linearisation which, locally to the equilibrium operating point, has similar dynamics to the plant, linear techniques may be used to resolve this local design task. Continuity is, therefore, maintained with established linear design techniques for which a considerable body of experience has been accumulated. While this traditional gain-scheduling approach is extremely successful

*Corresponding author. Tel.: +44-(0)141-548-2378; fax: +44(0)141-548-4203.

E-mail address: m.grimble@eee.strath.ac.uk (D.J. Leith). in most flight control applications (McLean, 1990), the trend is towards vehicle configurations where the conventional gain-scheduling conditions may not always be satisfied. Gain-scheduled controllers are traditionally designed on the basis of the dynamics relative to a family of trim conditions assuming that the airspeed is slowly varying. However, during aggressive manoeuvring, the vehicle may be far from equilibrium with rapidly varying airspeed (McLean, 1990, p. 523). In addition, the requirement to operate at high angles of attack can necessitate scheduling on rapidly varying quantities such as the instantaneous incidence angle (rather than, for example, conventional flap scheduling on averaged incidence, McLean, 1990, p. 523). It should be noted that scheduling on instantaneous incidence is wellknown to be problematical and is almost always avoided in classical scheduling arrangements (see, for example, Leith \& Leithead, 2000b). Specifically, in the example considered in this paper, a conventional gain-scheduling design approach fails to lead to a stabilising controller. There is, consequently, interest in the literature, towards alternative nonlinear control design approaches such as dynamic inversion (see, for example, Meyer, Su, \& Hunt, 1984; Tsourdos, Blumel, \& White, 1998). However, in addition to the robustness issues associated with 
controllers based on inversion, owing to the substantial body of experience which has been accumulated with gain-scheduling methods both with regard to meeting performance requirements and also such practical issues as safety certification, there is a strong incentive to retain the gain-scheduling approach.

Faced with these kind of issues (which are also relevant in many other applications), in recent years, a number of alternative approaches have been proposed which attempt to extend gain-scheduling methods including those based on local model networks and Takagi-Sugeno fuzzy models (see, for example, Johansen \& Murray-Smith, 1997; Hunt \& Johansen, 1997). However, these typically include off-equilibrium information at the cost of moving to nonlinear (especially affine) formulations and so lose the continuity with linear methods which is one of the principle advantages of classical gain-scheduling. Moreover, the requirement for some form of slow variation condition is often retained either directly or indirectly via assumptions implicit in the formulation (Leith \& Leithead, 1999a). Since the main source of these, and other, difficulties ultimately lies in the well-known limitations of classical equilibrium linearisation theory, it seems clear that the key to further progress lies in resolving the deficiencies of linearisation theory itself. It is precisely this issue which is addressed by the velocity-based analysis and design framework recently proposed in Leith and Leithead (1998a,b). This framework subsumes classical equilibrium linearisation theory and associates a linear system with every operating point (both equilibrium and off-equilibrium) of a nonlinear system, not just the equilibrium operating points. It thereby eliminates any restriction to near equilibrium operation while maintaining the continuity with linear methods as required. Moreover, it can be shown that the velocity-based approach does not inherently involve any slow variation requirement (Leith \& Leithead, 1999b). The literature relating to velocity-based methods is, at present, largely theoretical in nature, being concerned with establishing the fundamental properties of the velocity-based linearisation framework. The aims of the present paper are to bring together these theoretical results within the context of the nonlinear control design task, extend these results to achieve a coherent, soundly based gainscheduling design methodology and to demonstrate the effectiveness of the proposed methods in extending gainscheduling techniques to a highly nonlinear, rapidly varying nonlinear missile system operating far from equilibrium.

The paper is organised as follows: The control design task is introduced in Section 2. In Section 3, the velocitybased gain-scheduling framework is discussed and, in Section 4, a velocity-based gain-scheduled missile auto-pilot is designed. The results are summarised in Section 5.

\section{Control design task}

The missile lateral dynamics are (White, Tsourdos, \& Blumel, 1998; Tsourdos et al., 1998):

$$
\begin{aligned}
& \dot{r}=\frac{N}{I_{z}}=\frac{1}{2} I_{z}^{-1} \rho V_{0} S d C_{\mathrm{n}}, \\
& \dot{v}=\frac{Y}{m}-U r=\frac{1}{2} m^{-1} \rho V_{0} S C_{y}-U r, \\
& \eta_{y}=\dot{v}+U r,
\end{aligned}
$$

where $r$ is the yaw rate $(\mathrm{rad} / \mathrm{s}), v$ the lateral velocity $(\mathrm{m} / \mathrm{s}), \eta_{y}$ the lateral acceleration $\left(\mathrm{m} / \mathrm{s}^{2}\right), \delta$ the fin angle (rad). The model parameters are as follows: mass, $m=125 \mathrm{~kg}$; density, $\boldsymbol{\rho}=1.0 \mathrm{~kg} / \mathrm{m}^{-3}$; forward velocity, $V_{0}=1320 \mathrm{~m} / \mathrm{s} ;$ longitudinal velocity, $U=1000 \mathrm{~m} / \mathrm{s}$; reference area, $S=0.0314 \mathrm{~m}^{2}$; reference diameter, $d=0.2 \mathrm{~m}$; inertia, $I_{z}=67.5 \mathrm{~kg} \mathrm{~m}^{2}$. It is assumed that forward velocity is constant, that the longitudinal velocity is sufficiently large compared to the lateral velocity, that the total incidence angle may be accurately approximated by $v / V_{0}$ and that the roll angle is zero (skid-to-turn operation). Under these conditions, the aerodynamic coefficients $C_{\mathrm{n}}$ and $C_{y}$ corresponding to a Mach number of 4 are described by

$$
\begin{aligned}
C_{\mathrm{n}}= & \left(\frac{1}{2} d C_{\mathrm{n} r s}|v|+\frac{1}{2} d C_{\mathrm{n} r 0}\right) r-\left(\frac{X_{\mathrm{cps}} C_{y v s}}{d} v^{2}\right. \\
& \left.+\left(\frac{X_{\mathrm{cp} 0} C_{y v s}+X_{\mathrm{cps}} C_{y v 0}}{d}\right)|v|+\frac{X_{\mathrm{cpo}} C_{y v 0}}{d}\right) v \\
& +\left(S_{\mathrm{f}} V_{0} C_{y \delta s}|v|+S_{\mathrm{f}} V_{0} C_{y \delta 0}\right) \delta, \\
C_{y}= & \left(C_{y v 0}+C_{y v s}|v|\right) v+\left(V_{0} C_{y \delta 0}+V_{0} C_{y \delta s}|v|\right) \delta,
\end{aligned}
$$

where

$$
\begin{aligned}
& C_{\mathrm{n} r s}=8.6856, C_{\mathrm{n} r 0}=-616.4706, C_{y v s}=-2.6057, \\
& C_{y v 0}=-21.1176, C_{y \delta s}=0.0869, C_{y \delta 0}=-16.2118 \\
& X_{\mathrm{cp} 0}=0.1382, X_{\mathrm{cp} s}=0.0087, S_{\mathrm{f}}=-5.25 .
\end{aligned}
$$

The fin actuator, $A$, is modelled by linear dynamics with transfer function

$$
A(s)=\frac{360000}{s^{2}+600 s+360000} .
$$

The requirement is to achieve a nominal closed-loop acceleration response with rise time of less than $0.1 \mathrm{~s}$ and less that $20 \%$ overshoot. This requirement is to be satisfied over the whole operating envelope of $\pm 50 \mathrm{~g}$. Whilst this is, of course, not a complete performance specification, it is adequate for the present study (in particular, since consideration focuses on catering for the nonlinear aerodynamics, a simple actuator representation is employed and issues relating to measurement noise are not considered). 
The control design task for the agile missile is a demanding one owing to the strongly nonlinear nature of the dynamics, the large operating envelope with a requirement for rapid, aggressive manoeuvring, and the fast rise time specification (corresponding, in linear terms, to a bandwidth around twice that of the unaugmented system). The conventional approach is to adopt a gain-scheduling design methodology. The classical gain-scheduling design approach requires appropriate linearisations of the dynamics which approximate, locally to specific equilibrium flight conditions, the nonlinear dynamic behaviour of the missile. Let the equilibrium operating points be parameterised by $\pi$. The nonlinear dynamics of the missile (1) may then be approximated, locally to the specific equilibrium operating point $\pi_{\mathrm{o}}$, by the series expansion linearisation,

$\boldsymbol{\Delta} \dot{\boldsymbol{r}}=N_{r}\left(\pi_{\mathrm{o}}\right) \boldsymbol{\Delta} \boldsymbol{r}+N_{v}\left(\pi_{\mathrm{o}}\right) \boldsymbol{\Delta} v+N_{\delta}\left(\pi_{\mathrm{o}}\right) \boldsymbol{\Delta} \delta$,

$\Delta \dot{v}=-U_{0} \Delta r+Y_{v}\left(\pi_{\mathrm{o}}\right) \Delta v+Y_{\delta}\left(\pi_{\mathrm{o}}\right) \Delta \delta$,

$\Delta \eta_{y}=Y_{v}\left(\pi_{\mathrm{o}}\right) \Delta v+Y_{\delta}\left(\pi_{\mathrm{o}}\right) \Delta \delta$,

together with the input, output and state transformations,

$\Delta \delta=\delta-\delta_{0}, \quad r=r_{0}+\Delta r$,

$v=v_{0}+\Delta v, \quad \eta_{y}=\eta_{y_{0}}+\Delta \eta_{y}$,

where $\delta_{0}, r_{0}, v_{0}$ and $\eta_{y_{0}}$ are, respectively, the values of $\delta$, $r, v$ and $\eta_{y}$ at the equilibrium operating point $\pi_{\mathrm{o}}$ and

$$
\begin{aligned}
& N_{r}\left(\pi_{o}\right)=\left.\frac{1}{I_{z}} \frac{\partial N}{\partial r}\right|_{\pi_{o}}, \quad N_{v}\left(\pi_{o}\right)=\left.\frac{1}{I_{z}} \frac{\partial N}{\partial v}\right|_{\pi_{o}}, \\
& N_{\delta}\left(\pi_{\mathrm{o}}\right)=\left.\frac{1}{I_{\mathrm{z}}} \frac{\partial N}{\partial \delta}\right|_{\pi_{\circ}}, \quad Y_{v}\left(\pi_{\mathrm{o}}\right)=\left.\frac{1}{m} \frac{\partial Y}{\partial v}\right|_{\pi_{\circ}}, \\
& Y_{\delta}\left(\pi_{\mathrm{o}}\right)=\left.\frac{1}{m} \frac{\partial Y}{\partial \delta}\right|_{\pi_{\circ}} .
\end{aligned}
$$

Differentiating, (5) may be reformulated as

$$
\begin{aligned}
\boldsymbol{\Delta} \ddot{v} & -Y_{v}\left(\pi_{\mathrm{o}}\right) \boldsymbol{\Delta} \dot{v}+U_{0} N_{v}\left(\pi_{\mathrm{o}}\right) \boldsymbol{\Delta} v+U_{0} N_{r}\left(\pi_{\mathrm{o}}\right) \boldsymbol{\Delta} r \\
& =-U_{0} N_{\delta}\left(\pi_{\mathrm{o}}\right) \boldsymbol{\Delta} \delta+Y_{\delta}\left(\pi_{\mathrm{o}}\right) \boldsymbol{\Delta} \dot{\delta},
\end{aligned}
$$

which, following standard flight control practices and neglecting the direct coupling terms $Y_{\delta}$ may for control design purposes be simplified to

$$
\begin{aligned}
\Delta \ddot{v} & -Y_{v}\left(\pi_{\mathrm{o}}\right) \boldsymbol{\Delta} \dot{v}+U_{0} N_{v}\left(\pi_{\mathrm{o}}\right) \boldsymbol{\Delta} v+U_{0} N_{r}\left(\pi_{\mathrm{o}}\right) \Delta r \\
& =-U_{0} N_{\delta}\left(\pi_{\mathrm{o}}\right) \boldsymbol{\Delta} \delta,
\end{aligned}
$$

which is valid locally to the specific equilibrium operating point $\pi_{\mathrm{o}}$. Hence, the linearised plant dynamics at the equilibrium operating point $\pi_{\mathrm{o}}$ are described by (10) and (6) together with the input, output and state transformations (7). It should be noted that, whilst the input, output and state transformations (7) are different at every equilibrium operating point, the linearised dynamics, (10) and (6), are the same at equilibrium operating points for which $\pi$ equals $\pi_{\mathrm{o}}$. Hence, (10) and (6) define a linear dynamic family parameterised by $\pi$.

For each member of the linear family, (10) and (6), a linear controller is designed to meet the performance specification. A conventional cascaded inner-outer loop controller configuration is employed with a lateral velocity inner loop and an acceleration outer loop. The inner loop is designed to have sufficiently high bandwidth compared to the outer loop that the design of the two loops can be effectively de-coupled. Since the controllers are designed on the basis of the linearisation of the missile dynamics at trim flight conditions, the linear controllers act on perturbed quantities. Adopting what is perhaps the most widespread approach to constructing a nonlinear controller realisation, the scheduling variable is simply substituted into the linear controller family; that is, the gains of the controller are varied according to a suitable quantity which parameterises the equilibrium points (in the present example, lateral velocity is a natural choice) (see, for example, McLean, 1990, p. 298; Nichols, Reichert, \& Rugh, 1993; Blakelock, 1996) whilst relying on the feedback action to accommodate the input, output and state transformations. It should be noted that although the series expansion linearisations of the plant are employed in the design procedure, the corresponding local controller designs are frozen-scheduling variable linearisations of the resulting nonlinear controller.

This gain-scheduling approach is based on the assumptions that the system remains sufficiently near to equilibrium that the equilibrium linearisations are valid and that the rate of variation of the system from the vicinity of one equilibrium point to another is sufficiently slow. These assumptions are clearly not met in the present example (nor, indeed, in many realistic flight control applications). Whilst the controller is designed on the basis of the missile dynamics relative to trim conditions, the missile motion is not confined to small perturbations about trim but rather involves aggressive manoeuvring which takes the missile far from equilibrium. In addition, the scheduling variable, lateral velocity, is not a priori slowly varying relative to the dynamics of the controller; either with respect to the dynamics of the acceleration outer loop controller or the lateral velocity inner controller. These traditional gain-scheduling conditions are, however, conservative in nature. By nonlinear analysis of the resulting closed-loop system, the classical gain-scheduling approach (including the foregoing, on the face of it rather ad hoc, implementation technique) may lead to a soundly based controller design which is globally valid (not restricted to near equilibrium operation) provided that appropriate conditions are 
satisfied (Leith \& Leithead, 2000b). Namely, it is required that the aerodynamic moment and force are linear with respect to lateral velocity and fin angle and that $\partial N / \partial v, \partial N / \partial r, \partial N / \partial \delta, \partial Y / \partial v$ and $\partial Y / \partial \delta$ do not depend on the lateral velocity, $v$, or the fin angle, $\delta$; that is,

$$
\begin{aligned}
& N=\frac{\partial N}{\partial v}(\pi) v+\frac{\partial N}{\partial r}(\pi) r+\frac{\partial N}{\partial \delta}(\pi) \delta, \\
& Y=\frac{\partial Y}{\partial v}(\pi) v+\frac{\partial Y}{\partial \delta}(\pi) \delta,
\end{aligned}
$$

where the quantity, $\pi$, does not depend on the lateral velocity and fin angle. Also, it is required that the equilibrium operating points of the missile are parameterised by $\pi$ and that the rate of variation of $\pi$ is sufficiently slow that the nonlinear controller dynamics are insensitive to the choice of controller realisation. Evidently, the first two conditions are not satisfied in the present example and the third condition is unlikely to be satisfied owing to the rapid variation of the system. As might be expected, the gain-scheduled controller fails to achieve the performance specification even for relatively small acceleration demands (compared to the operating envelope of $\pm 50 \mathrm{~g}$ ) and, indeed, appears to de-stabilise the closed-loop system (the missile response, obtained using nonlinear simulations, to a step demand of $100 \mathrm{~m} / \mathrm{s}^{2}$, roughly $10 \mathrm{~g}$, in lateral acceleration is shown in Fig. 1).

Of course, the conventional gain-scheduling approach followed in the preceding discussion does not uniquely define the nonlinear controller. State-space representations of a linear controller which are related by a nonsingular linear state transformation are linearly equivalent; that is, they have the same transfer

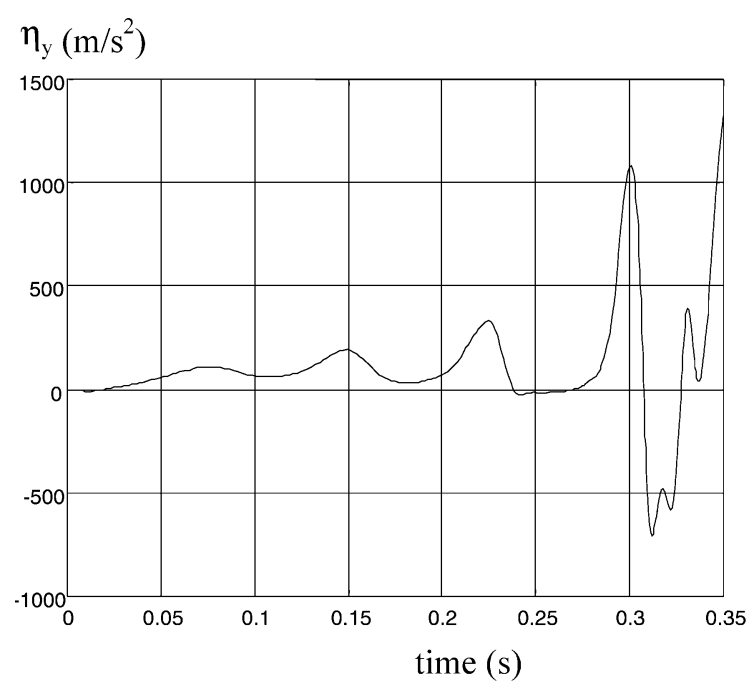

Fig. 1. Response of the closed-loop system to an acceleration step demand of $100 \mathrm{~m} / \mathrm{s}^{2}$ with the classical gain-scheduled controller. functions. Hence, each member of the family of linear controllers designed has infinitely many state-space representations. The selection of an appropriate gainscheduling controller realisation is considered by Lawrence and Rugh (1995) and Leith and Leithead (1998c). The utility of the former approach is, however, somewhat limited in general (Leith \& Leithead, 1998d, 1999c). In the latter approach, a controller realisation is sought which leads to the weakest slow variation requirement within the context of conventional gainscheduling, that is, on the basis of the plant dynamics relative to the equilibrium operating points. However, the slow variation requirement can, in general, be further weakened by exploiting knowledge of the plant dynamics at nonequilibrium operating points (Leith \& Leithead, 1998d). In the present example, the controller realisation is inadequate despite satisfying both the conditions proposed by Lawrence and Rugh (1995) and those considered by Leith and Leithead (1998c).

The requirement to design a gain-scheduled controller which achieves the performance specification remains to be achieved.

\section{Velocity-based gain-scheduling framework}

The velocity-based analysis and design framework, proposed recently in Leith and Leithead (1998b-d), associates a linearisation with every operating point of a nonlinear system, not just the equilibrium operating points. The approach thereby relaxes the restriction to near equilibrium operation whilst maintaining the continuity with linear methods which is a principle advantage of conventional gain-scheduling. Moreover, the velocity-based approach does not inherently involve a slow variation requirement. It therefore provides an appropriate framework within which to investigate the design of a gain-scheduled controller for the missile considered here.

Consider the nonlinear system

$\dot{\mathbf{x}}=\mathbf{F}(\mathbf{x}, \mathbf{r}), \quad \mathbf{y}=\mathbf{G}(\mathbf{x}, \mathbf{r})$,

which may be reformulated, without loss of generality, as

$\dot{\mathbf{x}}=\mathbf{A x}+\mathbf{B r}+\mathbf{f}(\rho), \quad \mathbf{y}=\mathbf{C} \mathbf{x}+\mathbf{D r}+\mathbf{g}(\rho)$,

where $\mathbf{x} \in \Re^{n}, \mathbf{r} \in \mathfrak{R}^{m}$ and $\mathbf{A}, \mathbf{B}, \mathbf{C}, \mathbf{D}$ are appropriately dimensioned constant matrices, $\mathbf{f}(\bullet)$ and $\mathbf{g}(\bullet)$ are nonlinear functions and $\rho(\mathbf{x}, \mathbf{r}) \in \mathfrak{R}^{q}, q \leqslant m+n$, embodies the nonlinear dependence of the dynamics on the state and input with $\nabla_{\mathbf{x}} \rho, \nabla_{\mathbf{r}} \rho$ constant. Trivially, this reformulation can always be achieved by letting $\rho=$ $\left[\mathbf{x}^{\mathrm{T}} \mathbf{r}^{\mathrm{T}}\right]^{\mathrm{T}}$, in which case $q=m+n$. However, the nonlinearity of the system is frequently dependent on only a subset of the states and inputs, in which case the dimension, $q$, of $\rho$ is less than $m+n$. It is emphasised 
that this scheduling variable, $\rho$, is quite distinct from the quantity, $\pi$, employed in classical gain-scheduling approaches. While $\pi$ is only required to parameterise the equilibrium points of a system, $\rho$ directly embodies the global nonlinear dependence of the dynamics. Of course, the class of systems, denoted extended local linear equivalence (ELLE) systems, for which a one-toone correspondence exists between $\rho$ and the locus of equilibrium points is of particular importance with regard to classical gain-scheduling techniques, see Leith and Leithead (1998b,c). Differentiating (13), an alternative representation of the nonlinear system is

$\dot{\mathbf{x}}=\mathbf{w}$,

$\dot{\mathbf{w}}=\mathbf{A}(\rho) \mathbf{w}+\mathbf{B}(\rho) \dot{\mathbf{r}}$,

$\dot{\mathbf{y}}=\mathbf{C}(\rho) \mathbf{w}+\mathbf{D}(\rho) \dot{\mathbf{r}}$,

where

$$
\begin{array}{ll}
\mathbf{A}(\rho)=\mathbf{A}+\nabla \mathbf{f}(\rho) \nabla_{\mathbf{x}} \rho, & \mathbf{B}(\rho)=\mathbf{B}+\nabla \mathrm{f}(\rho) \nabla_{\mathbf{r}} \rho, \\
\mathbf{C}(\rho)=\mathbf{C}+\nabla \mathbf{g}(\rho) \nabla_{\mathbf{x}} \rho, & \mathbf{D}(\rho)=\mathbf{D}+\nabla \mathbf{g}(\rho) \nabla_{\mathbf{r}} \rho .
\end{array}
$$

Dynamically, (14)-(16), with appropriate initial conditions, and (13) are equivalent (have the same solution, $\mathbf{x})$. When $\mathbf{w}=\mathbf{A x}+\mathbf{B r}+\mathbf{f}(\rho), \mathbf{y}=\mathbf{C x}+\mathbf{D r}+\mathbf{g}(\rho)$ is invertible so that $\mathbf{x}$ may be expressed as a function of $\mathbf{w}$, $\mathbf{r}$ and $\mathbf{y}$, then the transformation relating (14)-(16) to (13) is algebraic. The relationship between (14)-(16) and (13) is evidently direct. Moreover, the directness of the relationship extends rather more deeply than might initially be expected. Consider the linear system, obtained by "freezing" (14)-(16) at the operating point, $\left(\mathbf{x}_{1}, \mathbf{r}_{1}\right)$,

$\dot{\hat{\mathbf{x}}}=\hat{\mathbf{w}}$,

$\dot{\hat{\mathbf{w}}}=\mathbf{A}\left(\rho_{1}\right) \hat{\mathbf{w}}+\mathbf{B}\left(\rho_{1}\right) \dot{\mathbf{r}}$,

$\dot{\hat{\mathbf{y}}}=\mathbf{C}\left(\rho_{1}\right) \hat{\mathbf{w}}+\mathbf{D}\left(\rho_{1}\right) \dot{\mathbf{r}}$,

where $\rho_{1}=\rho\left(\mathbf{x}_{1}, \mathbf{r}_{1}\right)$. System (18)-(20) is referred to as the velocity-based linearisation of (13) associated with the operating point $\left(\mathbf{x}_{1}, \mathbf{r}_{1}\right)$. It may be shown that the solutions to the linear system (18)-(20) are an accurate approximation to the solutions of the nonlinear system, (13), locally to the operating point $\left(\mathbf{x}_{1}, \mathbf{r}_{1}\right)$. Furthermore, while the solution to an individual velocity-based linearisation is only a locally accurate approximate, there exists a velocity-based linearisation, (18)-(20), for every operating point $(\mathbf{x}, \mathbf{r})$ and thus a velocity-based linearisation family, with members defined by (18)-(20), can be associated with the nonlinear system, (13). The solutions to the members of the family of velocity-based linearisations may be pieced together to approximate the global solution to the nonlinear system (13) to an arbitrary degree of accuracy (Leith \& Leithead, 1998a). It is emphasised that, unlike conventional series expan- sion linearisation approaches, no restriction to near equilibrium operation is involved.

With regard to controller design, the velocity-based linearisation of the feedback combination of a plant and controller is simply the feedback combination of the velocity-based linearisations of the plant and controller (Leith \& Leithead, 1998c). The following velocity-based gain-scheduling design procedure is, therefore, suggested (Leith \& Leithead, 1998b):

1. Determine the velocity-based linearisation family associated with the nonlinear plant dynamics.

2. Based on the velocity-based linearisation family of the plant, determine the required velocity-based linearisation family of the controller such that the resulting closed-loop family achieves the performance requirements. Since each member of the plant family is linear, conventional linear design methods can be utilised to design each corresponding member of the controller family.

3. Implement a nonlinear controller with the velocitybased linearisation family designed at step 2. This step is discussed in detail in Leith and Leithead $(1998 b, c)$. When the controller contains integral action, the controller velocity-based linearisation family may be implemented directly.

This design procedure retains a divide and conquer approach and maintains the continuity with linear design methods which is an important feature of the conventional gain-scheduling approach. However, in contrast to the conventional gain-scheduling approach, the resulting nonlinear controller is valid throughout the operating envelope of the plant, not just in the vicinity of the equilibrium operating points. This extension is a direct consequence of employing the velocity-based linearisation framework rather than the conventional series expansion linearisation about an equilibrium operating point.

Since there are a continuum of operating points, the velocity-based linearisation family associated with a nonlinear system has infinitely many members. It should be noted that a similar issue also arises in classical gainscheduling approaches since there are, in general, a continuum of equilibrium points and so infinitely many equilibrium linearisations. Although a direct controller design based on the analytic formulation of the plant dynamics might be constructed in step 2 above, in traditional gain-scheduling approaches it is more usual to design controllers for a small number of representative equilibrium points and interpolate between the resulting point designs to obtain a fully populated controller family. A similar approach is also attractive within the velocity-based gain-scheduling framework, but with consideration extended to include off-equilibrium operating points in addition to equilibrium points. The requirement, not addressed in the existing 
literature, is to modify the above velocity-based design procedure to one based on a small number of operating points, that is, to an exact analogy of classical gainscheduling. It is addressed by bringing together the results in Leith and Leithead (1999a, b) as developed below.

A key issue in any approach based on a small number of operating points is the choice of interpolation strategy. Traditional gain-scheduling approaches typically place emphasis on interpolating control designs with little reference to the plant characteristics with the result that the interpolation approach often appears rather arbitrary. Within the velocity-based framework a more soundly based approach is suggested. Since the control design is based on the velocity-based linearisation of the plant, the natural requirement is to determine a (perhaps approximate) finite parameterisation of the plant family on which to base gain-scheduled control designs. This requirement leads to consideration of a blended multiple model representation of the plant linearisation family whereby the linearisations at a small number of representative operating points are blended together/interpolated between to produce an approximation to the exact linearisation family (Leith \& Leithead, 1999a). Letting $\left\{\left(\mathbf{x}_{j}, \mathbf{r}_{j}\right)\right\}$ denote the centre operating points upon which the blended family is based, the corresponding representation of the plant linearisation family is

$\dot{\tilde{\mathbf{x}}}=\tilde{\mathbf{w}}$,

$\dot{\tilde{\mathbf{w}}}=\left(\sum_{j} \mathbf{A}^{j} \mu_{j}(\tilde{\rho})\right) \tilde{\mathbf{w}}+\left(\sum_{j} \mathbf{B}^{j} \mu_{j}(\tilde{\rho})\right) \dot{\mathbf{r}}$,

$\dot{\tilde{\mathbf{y}}}=\left(\sum_{j} \mathbf{C}^{j} \mu_{j}(\tilde{\rho})^{j}\right) \tilde{\mathbf{w}}+\left(\sum_{j} \mathbf{D}^{j} \mu_{j}(\tilde{\rho})\right) \dot{\mathbf{r}}$,

$\tilde{\rho}=\nabla_{\mathbf{x}} \rho \tilde{\mathbf{x}}+\nabla_{\mathbf{r}} \rho \mathbf{r}$,

with $\quad \mathbf{A}^{j}=\mathbf{A}\left(\rho_{j}\right), \mathbf{B}^{j}=\mathbf{B}\left(\rho_{j}\right), \mathbf{C}^{j}=\mathbf{C}\left(\rho_{j}\right), \mathbf{D}^{j}=\mathbf{D}\left(\rho_{j}\right)$. Provided $\mu_{j}\left(\rho_{j}\right)$ is unity and $\mu_{k}\left(\rho_{j}\right), k \neq j$, is zero, the frozen form of the blended multiple model system at the operating point $\left(\tilde{\mathbf{x}}_{j}, \mathbf{r}_{j}\right)$ is precisely the velocity-based linearisation of the plant at $\left(\tilde{\mathbf{x}}_{j}, \mathbf{r}_{j}\right)$. At any other operating point, say at $\left(\tilde{\mathbf{x}}_{1}, \mathbf{r}_{1}\right),(21)$ is the approximation to the velocity-based linearisation, (14)-(16), obtained by interpolating, by means of the validity functions $\mu_{j}\left(\rho_{1}\right)$, between the velocity-based linearisations at the operating points, $\left(\tilde{\mathbf{x}}_{j}, \mathbf{r}_{j}\right)$. Provided (21) is a sufficiently accurate approximation to the exact plant family and/or the controller is sufficiently robust, a controller which achieves the performance objectives with these approximate dynamics is also guaranteed to achieve satisfactory performance when used with the exact plant dynamics (14)-(16). The blended representation, (21), of the plant linearisation family thus leads directly to a divide and conquer control design approach whereby a linear controller is designed for the plant linearisation associated with each of the operating points in $\left\{\left(\mathbf{x}_{j}, \mathbf{r}_{j}\right)\right\}$. These control designs are then interpolated, using the same weighting functions $\mu_{j}$ as the plant, to obtain the complete velocity-based linearisation family of the controller (Leith \& Leithead, 1999a). Note that, similar to classical gain-scheduling, control designs are only required for the small number of centre operating points $\left\{\left(\mathbf{x}_{j}, \mathbf{r}_{j}\right)\right\}$. However, in contrast to classical approaches, the interpolating scheme adopted is explicitly selected to capture the plant dynamics. The blended representation (21) is closely related to neuro-fuzzy modelling approaches including local model networks and TakagiSugeno fuzzy methods. However, in contrast to these methods, the velocity-based representation:

- Blends genuinely linear (not affine) models.

- The dynamics are directly related to the centre models: the solution to a velocity-based blended multiple model system, locally to a specific operating point, is described by the solution to the linear system obtained by "freezing" the blended multiple model system at the relevant operating point. The resulting frozen system is simply a weighted linear combination of the centre models. This holds for both plant and controller and provides the theoretical justification for using identical weighting functions in both families.

- A still stronger property is that the solution to the blended multiple model system, locally to a specific operating point, is under suitable conditions approximated by the weighted linear combination of the solutions to the centre models (Leith \& Leithead, 1999a). It follows, for example, that when the centre models exhibit uniform step responses, this will also hold globally.

It is the close relationship thereby guaranteed between the properties of the linearisations at the centre operating points and those at intervening operating points that provides a sound basis for the proposed divide and conquer design approach.

In addition to the choice of interpolation strategy, a second key issue is to ensure that the members of the controller family are, in an appropriate sense, compatible with one another. Again, a similar issue arises in classical gain-scheduling approaches. However, the theoretical understanding of this area is very poorly developed within the classical framework. In many applications the requirement is for approximately uniform performance across the operating envelope. Consider, therefore, selecting the controller velocity-based linearisations such that at every operating point the closed-loop combination of the plant and controller linearisations has, approximately, the same transfer function. The open-loop linearisation family then has 
a similar transfer function at every operating point. This is, however, not sufficient to ensure that the controller achieves uniform performance across the operating envelope; that is, approximately, inverts the plant dynamics. The transfer function only specifies the realisation of a linearisation to within a linear state transformation. It is, therefore, also necessary to ensure that the state-space realisations of the members of the controller linearisation family are suitably compatible with one another. When the rate of variation of a nonlinear system is sufficiently slow, it can be shown that the controller becomes insensitive to the choice of realisation (Leith \& Leithead, 2000b). However, in general, it is straightforward to confirm the closed-loop dynamics may be extremely sensitive to the choice of realisation. The issue of an appropriate choice of controller realisation (equivalently, an appropriate choice of state co-ordinates for each member of the controller linearisation family) remains to be fully addressed by current theory. However, relevant results do exist for the class of approximate (but arbitrarily accurate) inverting controllers (Leith \& Leithead, 1999b). Specifically, consider approximating the velocity-based plant dynamics (14)-(16) by

$\dot{\boldsymbol{x}}=\mathbf{w}$,

$\dot{\boldsymbol{w}}=\mathbf{A}(\rho) \mathbf{w}+\mathbf{B}(\rho) \dot{\mathbf{r}}$,

$\dot{\mathbf{y}}=\hat{\mathbf{C}}(\rho) \mathbf{w}+\hat{\mathbf{D}}(\rho) \dot{\mathbf{r}}$,

with $\hat{\mathbf{C}}(\rho)=\mathbf{C}(\rho)+\varepsilon_{\mathrm{C}}, \hat{\mathbf{D}}(\rho)=\varepsilon_{d} ; \varepsilon_{\mathrm{C}}, \varepsilon_{d}$, are constants with $\varepsilon_{d}$ nonsingular (for simplicity, it is assumed, without loss of generality, that $\mathbf{D}(\rho)$ is zero). A realisation of the velocity-based inverse of (22)-(24) is

$\dot{\mathbf{w}}^{\text {inv }}=\mathbf{A}^{\text {inv }}(\rho) \mathbf{w}^{\text {inv }}+\mathbf{B}^{\text {inv }}(\rho) \dot{\zeta}$,

$\dot{\mathbf{r}}=\mathbf{C}^{\text {inv }}(\rho) \mathbf{w}^{\text {inv }}+\mathbf{D}^{\text {inv }}(\rho) \dot{\zeta}$,

where

$\mathbf{A}^{\text {inv }}(\rho)=\mathbf{A}(\rho)-\mathbf{B}(\rho) \hat{\mathbf{D}}^{-1}(\rho) \hat{\mathbf{C}}(\rho)$,

$\mathbf{B}^{\text {inv }}(\rho)=-\mathbf{B}(\rho) \hat{\mathbf{D}}^{-1}(\rho)$,

$\mathbf{C}^{\text {inv }}(\rho)=\hat{\mathbf{D}}^{-1}(\rho) \hat{\mathbf{C}}(\rho)$,

$\mathbf{D}^{\text {inv }}(\rho)=\hat{\mathbf{D}}^{-1}(\rho)$.

The controller (25) is an exact inverse of (22)-(24) but an approximate inverse of the original system (14)-(16). In particular, the cascade combination of (14)-(16) and (25) leads to the dynamics:

$$
\begin{aligned}
& {\left[\begin{array}{c}
\dot{\mathbf{z}} \\
\dot{\mathbf{w}}^{\text {inv }}
\end{array}\right]=\left[\begin{array}{cc}
\mathbf{A}(v) & 0 \\
0 & \mathbf{A}^{\text {inv }}(v)
\end{array}\right]\left[\begin{array}{c}
\mathbf{z} \\
\mathbf{w}^{\text {inv }}
\end{array}\right]+\left[\begin{array}{c}
0 \\
\mathbf{B}^{\text {inv }}(v)
\end{array}\right] \dot{\zeta},} \\
& \dot{\mathbf{y}}=\left[\begin{array}{ll}
\hat{\mathbf{C}}(v) & -\hat{\mathbf{C}}(v)
\end{array}\right]\left[\begin{array}{c}
\mathbf{z} \\
\mathbf{w}^{\text {inv }}
\end{array}\right],
\end{aligned}
$$

where $\mathbf{z}=\mathbf{w}+\mathbf{w}^{\text {inv }}$. Stability of the cascade combination requires that the inverse system is bounded-input bounded-output stable (ensuring that the unobservable $\mathbf{w}^{\text {inv }}$ dynamics, corresponding to the zero dynamics of (22)-(24), are stable) and that the uncontrollable subsystem in (27) involving the state, $\mathbf{z}$, is asymptotically stable. It can then be shown (Leith \& Leithead, 1999b) that under appropriate conditions, $\lim _{\varepsilon_{C}, \varepsilon_{d} \rightarrow 0} \hat{\mathbf{C}}(v) \mathbf{w}^{\text {inv }}=$ $-\dot{\zeta}$, and that the approximate inverse (25) can therefore be made arbitrarily accurate with respect to the original system (14)-(16). The values of $\varepsilon_{d}$ and $\varepsilon_{C}$ used, while deriving the approximate inverse, are design parameters which determine the accuracy of the inversion achieved. Analogously, to linear pole-zero inversion of systems with relative degree greater than zero, the approximate inverse contains high frequency poles (more precisely, the VB linearisation contains high frequency poles) to ensure that it is realisable. Roughly speaking, $\varepsilon_{d}$ determines the frequency of the poles and $\varepsilon_{C}$ influences the damping with the inversion error tending to zero as $\varepsilon_{d}$ and $\varepsilon_{\mathrm{C}}$ tend to zero. Certain aspects of the plant dynamics, such as sharp resonances, may cause the inverting controller to be sensitive to plant uncertainty. Hence, more generally, the type of approximation (22)(24) employed may be modified to improve robustness by ameliorating any such aspects of the plant dynamics, albeit at the cost of inexact inversion of the original dynamics. When such an approach is adopted, the inversion theory is, in effect, being used to determine an appropriate controller realisation rather than to invert the system per se. The inverting controller obtained should be augmented by a pre-compensating fixed controller as required to meet the design requirements and including roll-off at high frequencies of the overall controller gain. When applied in the context of a blended representation, (21), of the plant, the inversion procedure resolves the issue of ensuring that the members of the controller family are compatible with one another. The inverting controller for the nonlinear plant is obtained by designing an inverting controller for the plant linearisation associated with each of the operating points in $\left\{\left(\mathbf{x}_{j}, \mathbf{r}_{j}\right)\right\}$ and blending these linear designs together as in (21). It is worthwhile noting that, provided the output equation of the plant is linear so that the $\mathbf{C}^{j}$ and $\mathbf{D}^{j}$ are all the same, the resulting nonlinear controller is

$$
\begin{aligned}
& \dot{\tilde{\mathbf{w}}}^{\text {inv }}=\sum_{j} \mu_{j}(\tilde{\rho})\left(\mathbf{A}_{j}-\mathbf{B}_{j} \hat{\mathbf{D}}^{-1} \hat{\mathbf{C}}\right) \tilde{\mathbf{w}}^{\text {inv }}-\sum_{j} \mu_{j}(\tilde{\rho}) \mathbf{B}_{j} \hat{\mathbf{D}}^{-1} \dot{\zeta} \\
& \dot{\mathbf{r}}=\hat{\mathbf{D}}^{-1} \hat{\mathbf{C}} \tilde{\mathbf{w}}^{\text {inv }}+\hat{\mathbf{D}}^{-1} \dot{\zeta}
\end{aligned}
$$

which is identical to the inverting controller obtained by applying (25) and (26) to (21).

To summarise, the analogy to classical gain-scheduling within the velocity-based framework consists of the 
foregoing design procedure with steps 1 and 2 modified as follows.

(1) Determine an appropriate velocity-based blended model for the nonlinear system. The blended model consists of a small number of velocitybased linearisations together with the interpolation functions to represent all the dynamics both on and off equilibrium.

(2a) Modify the velocity-based blended model as required to ensure invertibility, to reduce sensitivity to plant uncertainty and to reduce complexity.

(2b) Invert each velocity-based linearisation and blend together using the same interpolating functions as in (1).

The resulting controller is globally valid and involves no slow variation requirement.

\section{Velocity-based gain-scheduled missile auto-pilot}

Differentiating, the missile dynamics (1) may be reformulated in the velocity-based form:

$\dot{\mathbf{w}}=\mathbf{A}(\rho) \mathbf{w}+\mathbf{B}(\rho) \dot{\delta}, \quad\left[\begin{array}{c}\dot{v} \\ \dot{\eta}_{\mathrm{y}}\end{array}\right]=\mathbf{C}(\rho) \mathbf{w}+\mathbf{D}(\rho) \dot{\delta}$,

where

$\mathbf{w}=\left[\begin{array}{c}\dot{r} \\ \dot{v}\end{array}\right], \quad \mathbf{A}(\rho)=\left[\begin{array}{cc}\frac{1}{I_{\mathrm{z}}} \frac{\partial N}{\partial r}(v, r, \delta) & \frac{1}{I_{\mathrm{z}}} \frac{\partial N}{\partial v}(v, r, \delta) \\ -U & \frac{1}{m} \frac{\partial Y}{\partial v}(v, \delta)\end{array}\right]$,

$\mathbf{B}(\rho)=\left[\begin{array}{c}\frac{1}{I_{\mathrm{z}}} \frac{\partial N}{\partial \delta}(v, r, \delta) \\ \frac{1}{m} \frac{\partial Y}{\partial \delta}(v, \delta)\end{array}\right], \quad \mathbf{C}(\rho)=\left[\begin{array}{cc}0 & 1 \\ 0 & \frac{1}{m} \frac{\partial Y}{\partial v}(v, \delta)\end{array}\right]$,

$\mathbf{D}(\rho)=\left[\begin{array}{c}0 \\ \frac{1}{m} \frac{\partial Y}{\partial \delta}(v, \delta)\end{array}\right]$.

The velocity-based linearisation associated with an operating point is obtained by simply "freezing" (30) at the relevant operating point and the collection of such linearisations forms the velocity-based linearisation family. It can be seen, by inspection, that the scheduling variable, $\rho$, associated with this system is $\left[\begin{array}{lll}v & r & \delta\end{array}\right]^{\mathrm{T}}$. Note that that while $v, r$, and $\delta$ are related at equilibrium operating points, this is not the case at nonequilibrium operating points; that is, $v_{0}$ and $r_{0}$ are functions of $\delta$, but $v$ and $r$ in general are not functions of $\delta$ and so every triple $(v, r, \delta)$ does not correspond to a triple $\left(v_{0}(\delta), r_{0}(\delta), \delta\right)$. Hence, the velocity-based linearisation family contains members which do not correspond to any of those of the equilibrium linearisation family (and so which are not captured by any classical gainscheduling approach).

An inner/outer loop type of structure is adopted with a lateral velocity inner loop designed first and then enclosed within an acceleration outer loop. ${ }^{1}$ The inner loop is designed to have sufficiently higher bandwidth than the outer loop so that the design of the two loops can be effectively de-coupled. One immediate advantage of this approach is that the dynamics from fin angle to lateral velocity are minimum-phase (unlike the dynamics from fin to lateral acceleration which are non-minimumphase and so would hinder the control design). The specification and target loop dynamics remain the same as those for the conventional gain-scheduled controller discussed in Section 2.

\subsection{Inner-loop design}

For control design (but not analysis) purposes, the lateral velocity dynamics of the plant are approximated by the blended representation

$\dot{\hat{\mathbf{w}}}=\hat{\mathbf{A}}(v) \hat{\mathbf{w}}+\hat{\mathbf{B}}(v) \dot{\hat{\delta}}, \quad \hat{w}_{v}=\hat{\mathbf{C}} \hat{\mathbf{w}}+\hat{\mathbf{D}} \dot{\hat{\delta}}$,

with

$$
\begin{aligned}
& \hat{\mathbf{A}}(v)=\sum_{j=1}^{5} \mu_{j}(|v|) \mathbf{A}_{j}, \quad \hat{\mathbf{B}}(v)=\sum_{j=1}^{5} \mu_{j}(|v|) \mathbf{B}_{j}, \\
& \hat{\mathbf{C}}=\left[\begin{array}{ll}
\varepsilon_{\mathrm{C}} & 1
\end{array}\right], \quad \hat{\mathbf{D}}=\varepsilon_{d},
\end{aligned}
$$

where

$$
\begin{aligned}
& \hat{\mathbf{w}}=\left[\begin{array}{l}
\hat{w}_{r} \\
\hat{w}_{v}
\end{array}\right], \\
& \mathbf{A}_{1}=\mathbf{A}\left(\left[\begin{array}{l}
0 \\
0 \\
0
\end{array}\right]\right)=\left[\begin{array}{cc}
-3.38 & 0.62 \\
-1000 & -3.24
\end{array}\right], \\
& \mathbf{A}_{2}=\mathbf{A}\left(\left[\begin{array}{l}
10 \\
0 \\
0
\end{array}\right]\right)=\left[\begin{array}{cc}
-2.85 & 5.70 \\
-1000 & -11.88
\end{array}\right], \\
& \mathbf{A}_{3}=\mathbf{A}\left(\left[\begin{array}{c}
20 \\
0 \\
0
\end{array}\right]\right)=\left[\begin{array}{cc}
-2.32 & 15.37 \\
-1000 & -20.52
\end{array}\right],
\end{aligned}
$$

\footnotetext{
${ }^{1}$ In practice, the inner loop would be augmented with feedforward from the acceleration demand but this is not omitted here in order to focus on the capability of the design method to compensate for the aerodynamic nonlinearity.
} 
$\mathbf{A}_{4}=\mathbf{A}\left(\left[\begin{array}{c}40 \\ 0 \\ 0\end{array}\right]\right)=\left[\begin{array}{cc}-1.25 & 48.47 \\ -1000 & -37.79\end{array}\right]$,

$\mathbf{A}_{5}=\mathbf{A}\left(\left[\begin{array}{c}60 \\ 0 \\ 0\end{array}\right]\right)=\left[\begin{array}{cc}-0.18 & 99.92 \\ -1000 & -55.07\end{array}\right]$,

$\mathbf{B}_{1}=\mathbf{B}\left(\left[\begin{array}{l}0 \\ 0 \\ 0\end{array}\right]\right)=\left[\begin{array}{c}5502.8 \\ -2830.0\end{array}\right]$,

$\mathbf{B}_{2}=\mathbf{B}\left(\left[\begin{array}{c}10 \\ 0 \\ 0\end{array}\right]\right)=\left[\begin{array}{c}5166.8 \\ -2657.2\end{array}\right]$

$\mathbf{B}_{3}=\mathbf{B}\left(\left[\begin{array}{c}20 \\ 0 \\ 0\end{array}\right]\right)=\left[\begin{array}{c}4830.8 \\ -2484.4\end{array}\right]$,

$\mathbf{B}_{4}=\mathbf{B}\left(\left[\begin{array}{c}40 \\ 0 \\ 0\end{array}\right]\right)=\left[\begin{array}{c}4158.8 \\ -2138.8\end{array}\right]$,

$\mathbf{B}_{5}=\mathbf{B}\left(\left[\begin{array}{c}60 \\ 0 \\ 0\end{array}\right]\right)=\left[\begin{array}{c}3486.8 \\ -1793.2\end{array}\right]$.

Note, only the first centre operating point is an equilibrium point. This blended/interpolated representation involves three main modifications for control purposes: (i) the inclusion of perturbation terms $\varepsilon_{\mathrm{C}}$ and $\varepsilon_{d}$ to ensure a realisable velocity-based inverse, (ii) the coefficients in (30) which depend on yaw rate, $r$, and fin angle, $\delta$, are neglected (so that the coefficients in (32) vary only with lateral velocity, $v$ ) and (iii) the actuator dynamics are neglected by assuming that the input $\hat{\delta}$ to the actuator is identical to the actual fin angle $\delta$ (to avoid unnecessary inversion of the actuator dynamics by the controller). Values of 5 and 0.2 for, respectively, $\varepsilon_{\mathrm{C}}$ and $\varepsilon_{d}$ are found to lead to an inverse which achieves an adequate degree of accuracy. With regard to the blending, for simplicity, standard triangular membership functions, $\mu_{j}$, centred on lateral velocities of 0,10 , 20,40 and $60 \mathrm{~m} / \mathrm{s}$ are used with overlap occurring only between the weighting functions associated with neighbouring centres. This scheme corresponds to straightforward linear interpolation between the local models. Despite the very strongly nonlinear nature of the dynamics, only five local models are sufficient to capture the dynamics through the entire operating envelope up to around $\pm 60 \mathrm{~g}$. (The accuracy of the approximation can be assessed by comparing the transfer functions of the linearisations associated with (30) and (32) over a range of operating points. However, the plots are omitted here owing to space considerations.) This choice of centres, while effective, is not optimised and, consequently, the use of fewer models might be feasible.

An appropriate realisation of the velocity-based inverse of (32) (an approximate inverse of the actual plant dynamics, (30)) is

$$
\begin{aligned}
& \dot{\mathbf{w}}^{\text {inv }}=\mathbf{A}^{\text {inv }}(v) \mathbf{w}^{\text {inv }}+\mathbf{B}^{\text {inv }}(v) \dot{\zeta}, \\
& \dot{\hat{\delta}}=\mathbf{C}^{\text {inv }} \mathbf{w}^{\text {inv }}+\mathbf{D}^{\text {inv } \dot{\zeta},}
\end{aligned}
$$

where

$$
\begin{aligned}
& \mathbf{A}^{\text {inv }}(v)=\sum_{j=1}^{5} \mu_{j}(|v|)\left(\mathbf{A}_{j}-\mathbf{B}_{j} \hat{\mathbf{D}}^{-1} \hat{\mathbf{C}}\right), \\
& \mathbf{B}^{\text {inv }}(v)=-\sum_{j=1}^{5} \mu_{j}(|v|) \mathbf{B}_{j} \hat{\mathbf{D}}^{-1} \\
& \mathbf{C}^{\text {inv }}=\hat{\mathbf{D}}^{-1} \hat{\mathbf{C}} \\
& \mathbf{D}^{\text {inv }}=\hat{\mathbf{D}}^{-1} .
\end{aligned}
$$

Note that $\hat{\mathbf{C}}, \hat{\mathbf{D}}$ are constant so that (35) is an exact inverse for (32). The nonlinear controller dynamics, (35), are augmented by the linear controller, $C_{i}$, consisting simply of integral action. The gain, $K_{i}$, of the integrator is selected such that the inner loop cross-over frequency is around $130 \mathrm{rad} / \mathrm{s}$. The architecture of the overall inner-loop controller design is shown in Fig. 2a; the uniform cross-over frequency of the resulting open-loop dynamics is clearly evident from Fig. 3. The velocitybased formulation (35) of the nonlinear controller dynamics cannot be directly implemented owing to the derivative action at the input (see Fig. 2a). However, owing to the integral action in the linear element of the controller dynamics, $C_{i}$, the controller structure in Fig. 2a may be equivalently reformulated as in Fig. 2 b. The realisation in Fig. $2 b$ is now in a form suitable for implementation. Note that a direct link is maintained between the gains in the controller implementation and the gains in the original (nonimplementable) velocitybased formulation.

\subsection{Outer-loop design}

In velocity-based form, the lateral acceleration is related to lateral velocity and fin angle by

$\dot{\eta}_{\mathrm{y}}=\frac{1}{m} \frac{\partial Y}{\partial v}(v, \delta) w_{\mathrm{v}}+\frac{1}{m} \frac{\partial Y}{\partial \delta}(v, \delta) \dot{\delta}$,

with $w_{v}=\dot{v}$. Owing to the relatively high bandwidth of the inner loop, the lateral velocity may (for design, but 


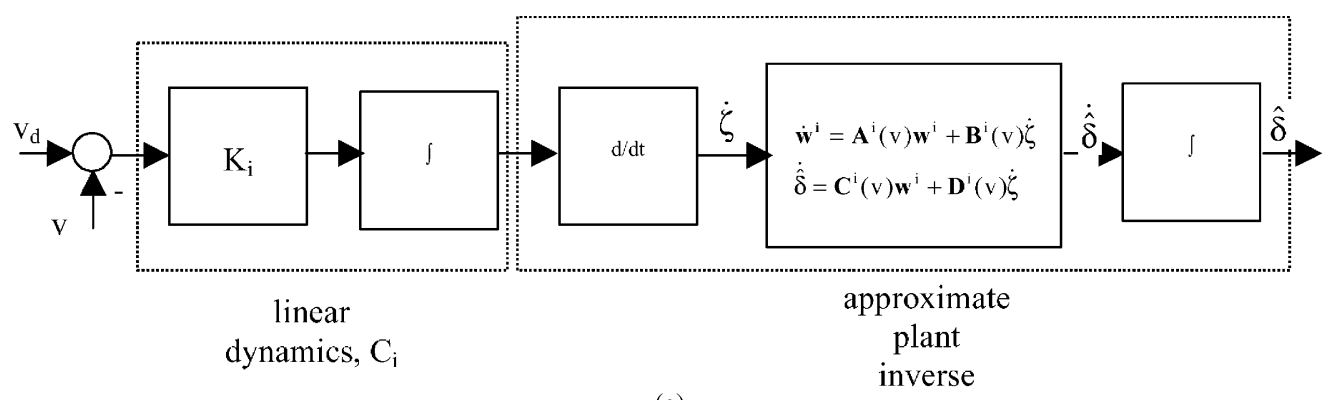

(a)

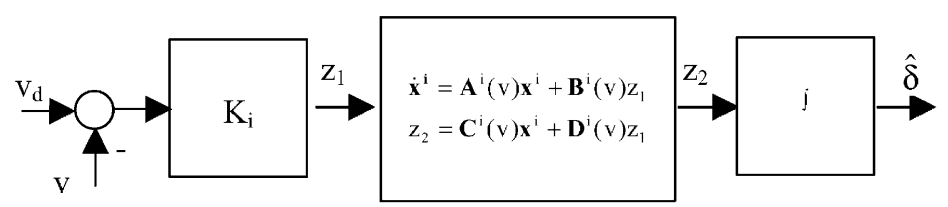

(b)

Fig. 2. Alternative formulations of inner-loop controller.
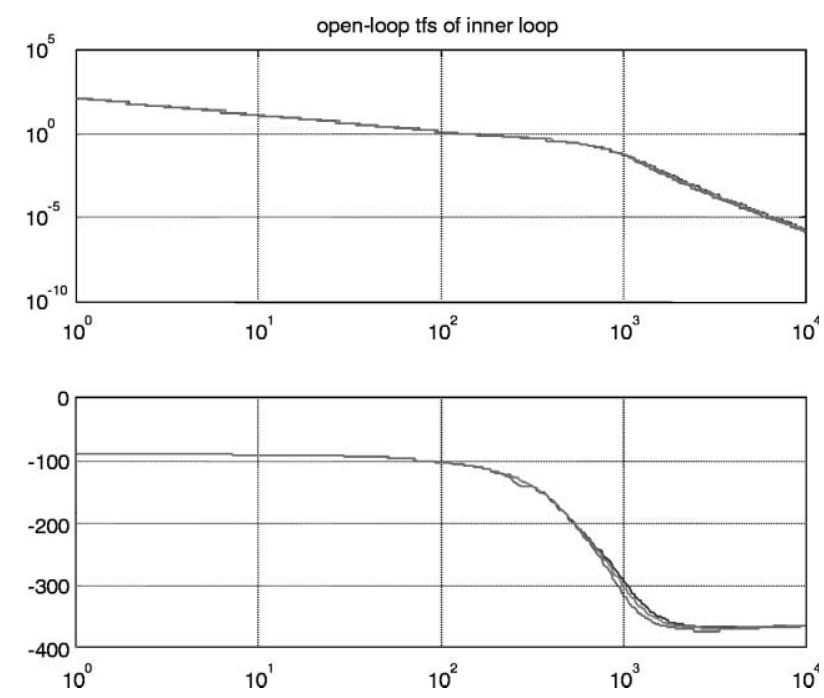

Fig. 3. Transfer functions of open-loop velocity-based linearisations of inner loop at lateral velocities of 0,20 and $50 \mathrm{~m} / \mathrm{s}$ (corresponding roughly to lateral accelerations of 0,20 and $50 \mathrm{~g}$ ).

not analysis, purposes), be considered effectively equal to the lateral velocity demand input to the inner-loop controller. Following standard flight control practice to modify the dynamics by neglecting the direct coupling term in (36) (with which the nonminimum phase dynamics are associated), the gain relating velocity to acceleration is approximately $(1 / m)(\partial Y / \partial v)(v, \delta)$. However, in addition to the expected gain mismatch at fast time-scales (high frequencies) due to neglecting the nonminimum phase zero in this approximation, there is also a mismatch in gain at long time-scales (low frequencies) when the direct coupling term is neglected: the mismatch at low frequency can be observed in the Bode plots for the velocity-based linearisations shown in
Fig. 4. Considering this effect in more detail, let $P$ and $P_{\mathrm{C}}$ denote, respectively, the velocity-based plant and inner-loop dynamic operators defined by

$w_{v}=P \dot{\delta}, w_{v}=P_{\mathrm{C}} \dot{v}_{d}$

It follows that

$\dot{\eta}_{y}=\left(\frac{1}{m} \frac{\partial Y}{\partial v}(v, \delta)+\frac{1}{m} \frac{\partial Y}{\partial \delta}(v, \delta) P^{-1}\right) P_{\mathrm{C}} \dot{v}_{d}$.

Since the bandwidth of both $P$ and $P_{\mathrm{C}}$ are considerably greater than the required bandwidth of the outer-loop, in (38) $P$ and $P_{\mathrm{C}}$ may be approximated by their steadystate gains yielding

$$
\begin{aligned}
& \dot{\eta}_{y} \approx\left(\frac{1}{m} \frac{\partial Y}{\partial v}(v, \delta)-\frac{1}{m} \frac{\partial Y}{\partial \delta}(v, \delta)\right. \\
& \left.\times \frac{\frac{U}{I_{z}} \frac{\partial N}{\partial v}(v, \delta)+\frac{1}{I_{z} m} \frac{\partial N}{\partial r}(v, \delta) \frac{\partial Y}{\partial v}(v, \delta)}{\frac{U}{I_{z}} \frac{\partial N}{\partial \delta}(v, \delta)+\frac{1}{I_{z} m} \frac{\partial N}{\partial r}(v, \delta) \frac{\partial Y}{\partial \delta}(v, \delta)}\right) \dot{v}_{d} .
\end{aligned}
$$

Hence, for control design purposes the dynamics relating velocity demand to lateral acceleration are approximated by the blended representation:

$\dot{\eta}_{y} \approx\left(\sum_{j=1}^{7} \mu_{j}(|v|) g_{j}\right) w_{v}$

with $g_{1}=-2.91, g_{2}=-6.19, g_{3}=-8.89, g_{4}=-12.51$; $g_{5}=-12.81 ; g_{6}=-9.41 ; g_{7}=-3.69$ and triangular weighting functions, $\mu_{j}$ centred on lateral velocities of $0,5,10,20,40,50$ and $60 \mathrm{~m} / \mathrm{s}$. Since the dependence of the aerodynamic derivatives on fin angle, $\delta$, is weak, it is neglected in (40) to simplify the scheduling arrangements. An outer-loop controller possessing the structure 

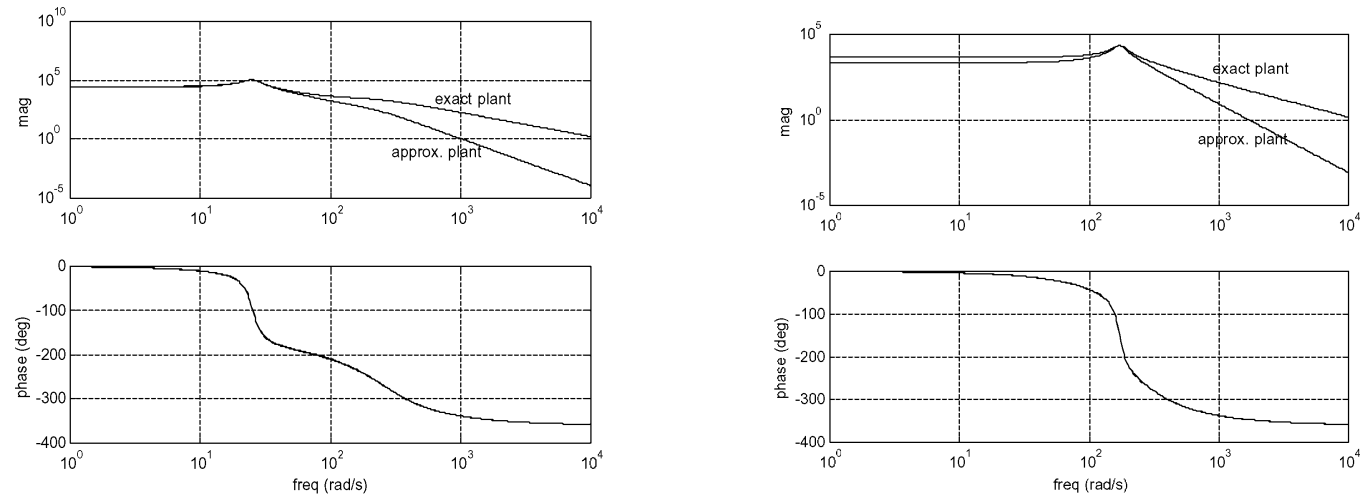

(a) $0 \mathrm{~m} / \mathrm{s}$

(b) $30 \mathrm{~m} / \mathrm{s}$
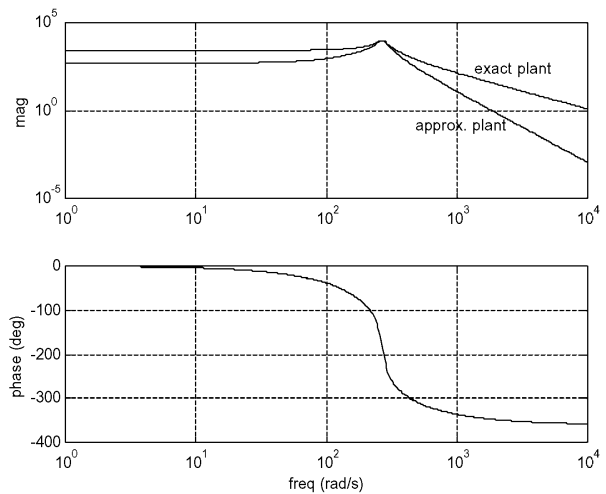

(c) $50 \mathrm{~m} / \mathrm{s}$

Fig. 4. Comparison of exact plant dynamics (relating acceleration to inner loop demand, $v_{\mathrm{d}}$ ) and nonminimum phase approximation.

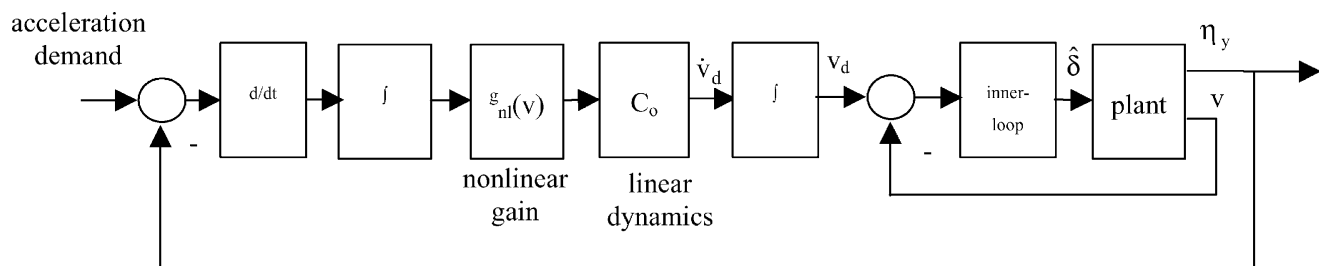

(a)

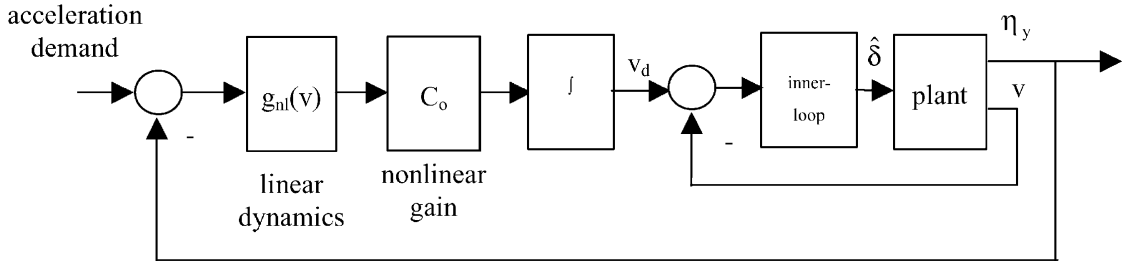

(b)

Fig. 5. Alternative formulations of outer-loop controller.

shown in Fig. 5a is therefore adopted, with nonlinear control gain $g_{\mathrm{nl}}$ satisfying

$g_{\mathrm{nl}}(v)=\left(\sum_{j=1}^{7} \mu_{j}(|v|) g_{j}^{-1}\right)$.

The nonlinear gain, $g_{\mathrm{nl}}$, is augmented with linear controller dynamics consisting of integral action com- bined with a low-pass filter, $C_{0}$, with transfer function, $1 /(0.02 s+1)$, for enhanced roll-off at high frequencies. The gain, $K_{0}$, of the integrator is selected such that the outer-loop bandwidth is around $20 \mathrm{rad} / \mathrm{s}$. Bode plots of the transfer functions of the velocity-based linearisations of the open outer-loop system are shown in Fig. 6. (It should be noted that these plots are for the exact system, including the dynamics of the inner control loop and 
with acceleration dynamics (36), rather than for the approximate system, neglecting the inner-loop dynamics and with acceleration dynamics (40), used for outer-loop control design purposes.) The velocity-based outer-loop controller realisation in Fig. 5a is not implementable owing to the derivative action. However, as discussed previously for the inner-loop controller, owing to the presence of integral action the outer-loop controller can be equivalently reformulated as the implementable form shown in Fig. $5 b$.

\subsection{Assessment of robust performance and stability}

Typical acceleration step responses with the velocitybased gain-scheduled controller are shown in Fig. 7. It can be seen that, in contrast to the previous classical gain-scheduled control design, the performance require-
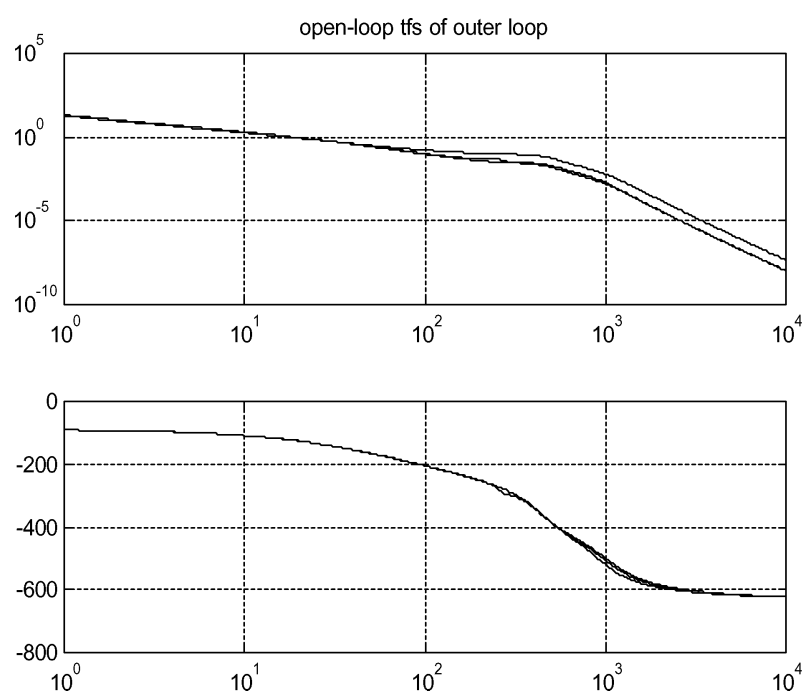

Fig. 6. Transfer functions of open-loop velocity-based linearisations of outer loop at lateral velocities of 0,20 and $50 \mathrm{~m} / \mathrm{s}$ (corresponding roughly to 0,20 and $50 \mathrm{~g}$ ). ments are indeed satisfied, under nominal conditions, over the full operating envelope $( \pm 50 \mathrm{~g})$. While performance under nominal conditions is necessary it is not, of course, sufficient. The requirement is to achieve robust stability and performance. This is particularly important in the guided weapons context where the dynamics are subject to very considerable uncertainty and a wide range of operating environments must be tolerated. In particular, a significant component of the uncertainty in flight vehicles is often highly structured in nature and, in the present application, the primary requirement is for robustness with respect to variations in the missile aerodynamic parameters. Methods for analysing the robustness of linear systems to parametric uncertainty have been the subject of a great deal of research in recent years and are relatively well developed. In contrast, the parametric robustness analysis of nonlinear, and in particular gain-scheduled controllers have received almost no attention and remain immature. However, by exploiting the continuity with linear methods which is provided by the velocity-based framework, the wealth of linear analysis methods can be brought to bear directly on nonlinear systems.

A linear system is said to be $\Gamma$-stable when the zeros of its characteristic polynomial lie inside a region $\Gamma$ of the complex left-hand plane (Ackermann, 1994). When $\Gamma$ coincides with the open left half-plane, then $\Gamma$ stability is equivalent to conventional Hurwitz stability. Applying the Kharitonov's theorem (see, for example, Weinmann, 1991), it can be shown that the members of the velocity-based linearisation family of the closed-loop missile system are stable for rather large variations in the missile aerodynamic parameters. Specifically, under worst case conditions the closed-loop linearisations are guaranteed Hurwitz stable over the range of parameters corresponding to simultaneous variations of $\pm 20 \%$ in $C_{y z 0}, C_{y z s}$ and $V_{0}, \pm 80 \%$ in $C_{y v 0}, C_{y v s}, X_{\mathrm{cp} 0}$ and $X_{\mathrm{cp} s}$, and $\pm 60 \%$ in $C_{n r 0}$ and $C_{n r s}$. Fig. 8 shows the worst case stability test results. The foregoing results relate to

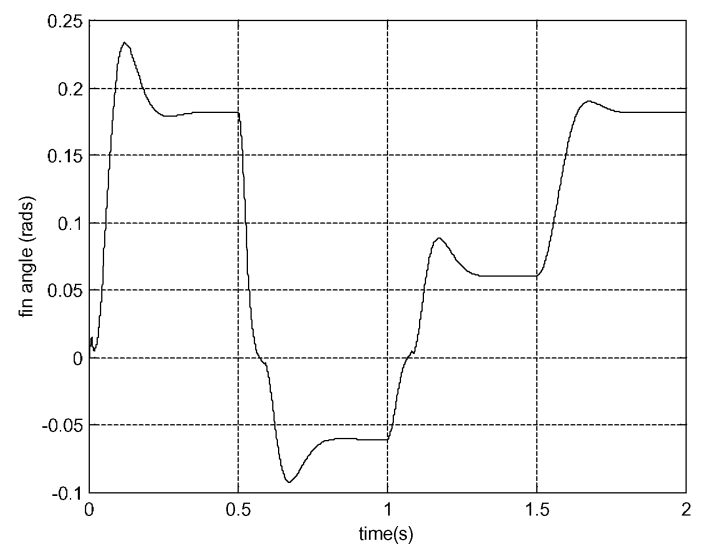

Fig. 7. Step responses with nominal plant and velocity-based gain-scheduled controller. 
robust Hurwitz stability. Robust performance can be analysed by additional requirements on the root locations as specified by a region $\Gamma$ in the complex $s$-plane (Ackermann, 1994). The $\Gamma$-region can be obtained by setting specific performance criteria such as peak overshoot (or damping) and settling time. For perturbations of $\pm 20 \%$ in the aerodynamic parameters, $\Gamma$-stability test results for the members of the closedloop velocity-based linearisation family are shown in Fig. 9 ( $\Gamma$ corresponding to a peak overshoot requirement of $M_{\mathrm{p}} \leqslant 30 \%$ ), Fig. 10 ( $\Gamma$ corresponding to a settling time requirement of $\left.t_{\mathrm{s}} \leqslant 0.2 \mathrm{~s}\right)$ and Fig. $11(\Gamma$ corresponding to $M_{\mathrm{p}} \leqslant 30 \%$ and $t_{\mathrm{s}} \leqslant 0.2 \mathrm{~s}$ ). These robust stability and performance results apply to the members of the velocity-based linearisation family of the closedloop system. Robust stability and performance of the linearisations need not, of course, imply the corresponding robust stability and performance of the associated nonlinear system (current theoretical results guarantee such a relationship only under appropriate slow variation conditions (Leith \& Leithead, 1998a). Nevertheless, simulation results confirm the robust stability and

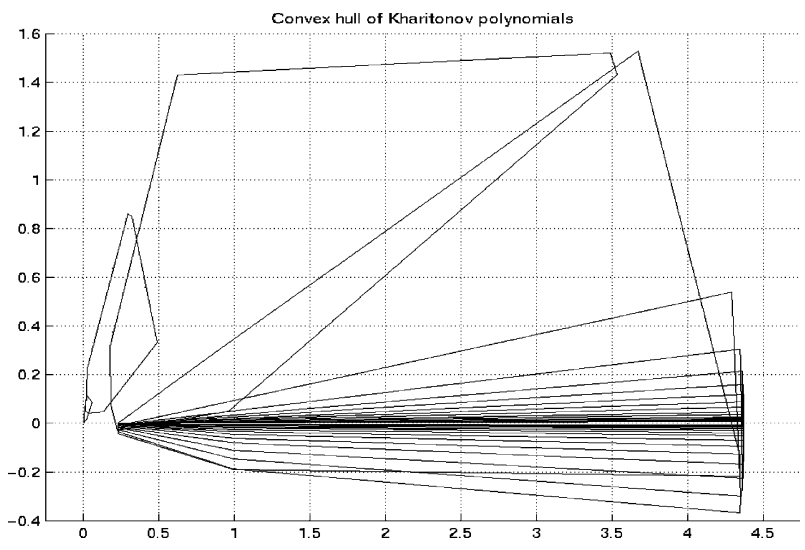

Fig. 8. Graphical robust Hurwitz stability test.

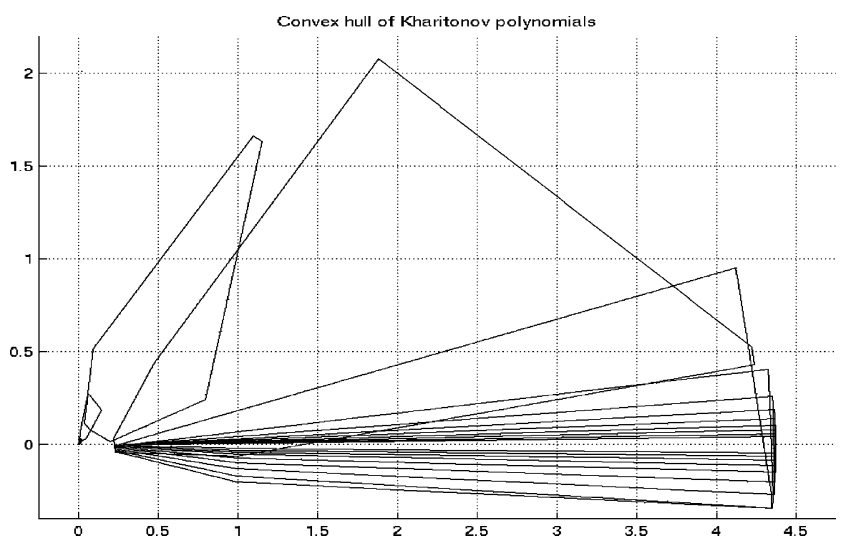

Fig. 9. Graphical robust $\Gamma$-stability test $\left(M_{\mathrm{p}} \leqslant 30 \%\right)$. performance of the nonlinear system as indicated by the foregoing analysis; for example, the step responses for independent variations of $\pm 20 \%$ in the aerodynamic parameters are shown in Fig. 12.

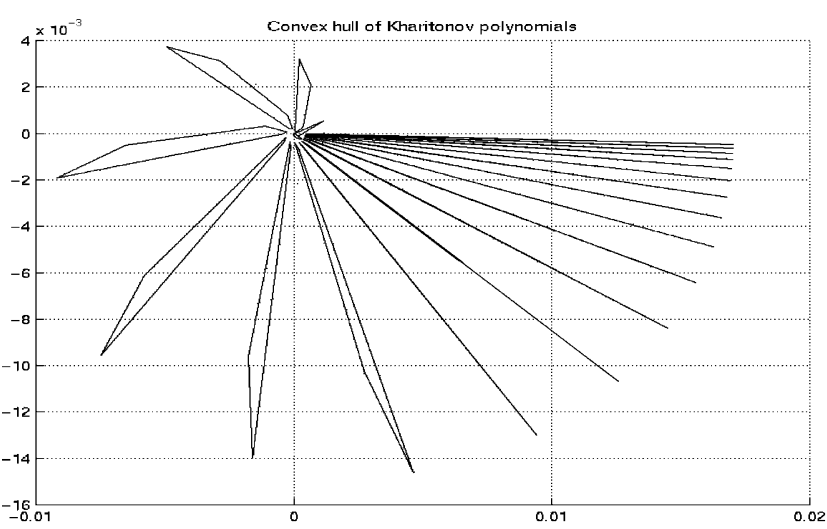

Fig. 10. Graphical robust $\Gamma$-stability test $\left(t_{s} \leqslant 0.2 \mathrm{~s}\right)$.

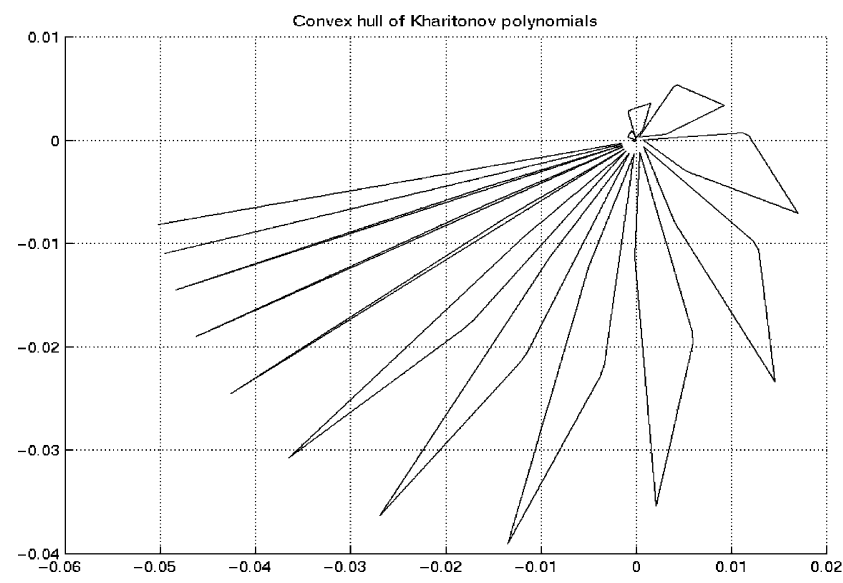

Fig. 11. Graphical robust $\Gamma$-stability test $\left(M_{\mathrm{p}} \leqslant 30 \%, t_{s} \leqslant 0.2 \mathrm{~s}\right)$.

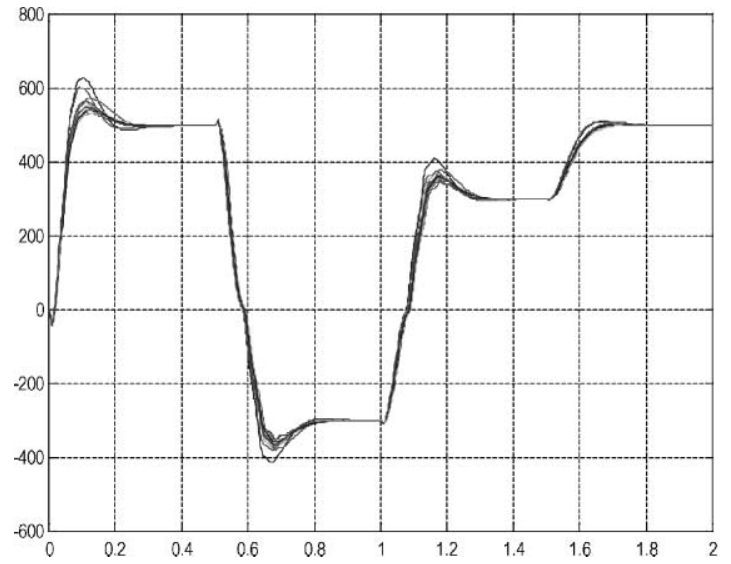

Fig. 12. Step responses of perturbed $( \pm 25 \%$ variations in aerodynamic parameters) plant with velocity-based gain-scheduled controller. 


\section{Summary and conclusions}

In this paper, a modern gain-scheduling methodology is proposed which exploits recently developed velocitybased techniques to resolve many of the deficiencies of classical gain-scheduling approaches (restriction to near equilibrium operation and to slow rate of variation) and to address a number of outstanding, yet fundamental, issues in gain-scheduling design (including the interpolation strategy and choice of controller realisation). This is achieved while maintaining a close analogy with classical gain-scheduling, including continuity with linear methods and an open design framework (any linear synthesis approach may be used) which directly supports divide and conquer design strategies. The formulation of this design framework is based upon a number of fundamental theoretical results which are brought together and extended to establish a coherent, and soundly based, methodology for nonlinear gainscheduling design. Particular issues addressed here include:

- Inclusion of dynamic information at off-equilibrium operating points as well as at equilibrium points. This addresses the key limitation of classical approaches and creates the opportunity for designing controllers which are globally valid.

- Support for divide and conquer design. Interpolation between linear control designs is formulated as a modelling task which exploits recent developments in blended multiple model representations of nonlinear systems. The utility of this approach is demonstrated by the support provided for divide and conquer design of global dynamic inversion controllers.

- Selection of controller realisation. The transfer function only specifies the realisation of a linearisation to within a linear state transformation. It is, therefore, also necessary to ensure that the state-space realisations of the members of the controller linearisation family are suitably compatible with one another. Recent results in (approximate) dynamic inversion theory are used to develop a soundly based approach for the selection of an appropriate choice of controller realisation (equivalently, an appropriate choice of state coordinates for each member of the controller linearisation family).

It is emphasised that the resulting controllers are globally valid and involve no inherent slow variation requirement. The application of velocity-based gainscheduling techniques is demonstrated in a demanding, highly nonlinear, missile control design task. Scheduling on instantaneous incidence (a rapidly varying quantity) is well-known to lead to considerable difficulties with classical gain-scheduling methods. It is shown that the methods proposed here can, however, be used to successfully design an effective and robust gain-scheduled controller.

\section{Acknowledgements}

D.J. Leith acknowledges the generous support provided by the Royal Society for the work presented.

\section{References}

Ackermann, J. (1994). Robust control, systems with uncertain physical parameters. London: Springer.

Blakelock, J. (1996). Automatic control of aircraft and missiles. New York: Wiley.

Hunt, K. J., \& Johansen, T. A. (1997). Design and analysis of gainscheduled control using local controller networks. International Journal of Control, 66, 619-651.

Johansen, T. A., \& Murray-Smith, R. (1997). The operating regime approach to nonlinear modelling and Control. In R. MurraySmith, \& T. A. Johansen (Eds.), Multiple model approaches to modelling \& control. London: Taylor \& Francis.

Lawrence, D. A., \& Rugh, W. J. (1995). Gain scheduling dynamic linear controllers for a nonlinear plant. Automatica, 31, 381-390.

Leith, D. J., \& Leithead, W. E. (1998a). Gain-scheduled \& nonlinear systems: Dynamic analysis by velocity-based linearisation families. International Journal of Control, 70, 289-317.

Leith, D. J., \& Leithead, W. E. (1998b). Gain-scheduled controller design: An analytic framework directly incorporating non-equilibrium plant dynamics. International Journal of Control, 70, 249-269.

Leith, D. J., \& Leithead, W. E. (1998c). Appropriate realisation of MIMO gain scheduled controllers. International Journal of Control, 70, 13-50.

Leith, D. J., \& Leithead, W. E. (1998d). Comments on "Gain scheduling dynamic linear controllers for a nonlinear plant". Automatica, 34, 1041-1043.

Leith, D. J., \& Leithead, W. E. (1999a). Analytic framework for blended multiple model systems using linear local models. International Journal of Control, 72, 605-619.

Leith, D. J., \& Leithead, W. E. (1999b). Input-output linearisation by velocity-based gain-scheduling. International Journal of Control, 72, 229-246.

Leith, D. J., \& Leithead, W. E. (1999c). Further comments on "Gain scheduling dynamic linear controllers for a nonlinear plant". Automatica, 36, 173-174.

Leith, D. J.,\& Leithead, W. E. (2000a). Survey of gain-scheduling analysis \& design. International Journal of Control, in preparation.

Leith, D. J., \& Leithead, W. E. (2000b). Gain-scheduled control: Relaxing slow variation requirements by velocity-based design. Journal of Guidance, Control \& Dynamics, to appear.

Mclean, D. (1990). Automatic flight control systems. London: PrenticeHall.

Meyer, G., Su, R., \& Hunt, L. R. (1984). Application of nonlinear transformations to automatic flight control. Automatica, 20, 103-107.

Nichols, R. A., Reichert, R. T., \& Rugh, W. J. (1993). Gain-scheduling H-Infinity controllers: a flight control example. IEEE Transactions on Control Systems Technology, 1, 69-78. 
Tsourdos, A., Blumel, A., \& White, B. A. (1998). Trajectory control of a nonlinear homing missile. Proceedings of the 14th IFAC symposium on automatic control in aerospace, August 1998, Korea (pp. 118-123).

Weinmann, A. (1991). Uncertain models and robust control. Wien: Springer.
White, B. A., Tsourdos, A., \& Blumel, A. (1998). Lateral acceleration control design of a nonlinear homing missile. Proceedings of the 4th IFAC nonlinear control systems design symposium, July 1998, The Netherlands (pp. 707-712). 